\title{
Jung's type theorem for polynomial transformations of $\mathbb{C}^{2}$
}

\author{
by SŁAWomir KoŁodziej (Kraków)
}

Abstract. We prove that among counterexamples to the Jacobian Conjecture, if there are any, we can find one of lowest degree, the coordinates of which have the form $x^{m} y^{n}+$ terms of degree $<m+n$.

Introduction. In this note we prove the following

Theorem. Let $\Phi=(f, g): \mathbb{C}^{2}(x, y) \rightarrow \mathbb{C}^{2}(z, w)$ be a polynomial mapping of degree $m>1$ with constant (non-zero) Jacobian and let

$$
f=\sum_{j=0}^{m} f_{j}, \quad \operatorname{deg} f_{j}=j,
$$

be the expansion of $f$ into homogeneous polynomials. If the set $\left\{f_{m}=0\right\}$ is a complex line then there exists a polynomial automorphism $\Psi$ such that $\operatorname{deg} \Phi \circ \Psi<m=\operatorname{deg} \Phi$.

To give a context for this result we recall the famous Jacobian Conjecture [5] (see also [2], [7], [8]) saying that any polynomial transformation of $\mathbb{C}^{n}$ which has constant non-zero Jacobian is an automorphism. The theorem implies that if there exist counterexamples to the conjecture in $\mathbb{C}^{2}$ then those of the lowest degree among them fail to satisfy our assumption on the set $\left\{f_{m}=\right.$ $0\}$ (it is known [1], [6] that this set contains at most two complex lines).

As long as the Jacobian Conjecture is not proved the present theorem generalizes Jung's theorem [3]:

Any polynomial automorphism of $\mathbb{C}^{2}$ can be represented by means of a finite superposition of linear and triangular transformations defined by $z=x+c y^{m}, w=y$, where $c$ is a constant and $m$ is a positive integer.

Indeed, polynomial automorphisms satisfy the assumptions of our theorem and $\Psi$ from the statement is in fact a superposition of linear and triangular mappings.

1991 Mathematics Subject Classification: Primary 32N05. 
The proof of the theorem. After making a linear transformation in $\mathbb{C}^{2}(z, w)$ (resp. $\left.\mathbb{C}^{2}(x, y)\right)$ we may assume that $\operatorname{deg} g=\operatorname{deg} f=m$ (resp. $\left.f(x, y)=y^{m}+\sum_{j+k<m} c_{j k} x^{j} y^{k}\right)$. Set

$$
\frac{\alpha}{\beta}=\max \left\{\frac{j}{m-k}: c_{j k} \neq 0\right\},
$$

$\alpha, \beta$ coprime positive integers. It is clear that $0<\alpha / \beta<1$. Write $f$ in yet another form:

$$
f=\widehat{f}+f_{1}, \quad \text { where } \widehat{f}(x, y)=y^{m} W\left(x^{\alpha} / y^{\beta}\right),
$$

$W$ a polynomial of one variable $x^{\alpha} / y^{\beta}, W(0) \neq 0$, and

$$
f_{1}=\sum_{j /(m-k)<\alpha / \beta} c_{j k} x^{j} y^{k} .
$$

The Jacobian condition implies that if $\widehat{g}$ is defined in the same way as $\widehat{f}$ then

$$
\widehat{g}=\text { const. } \widehat{f} .
$$

Indeed, the Jacobian of $(\widehat{f}, \widehat{g})$ must be zero since $\widehat{f}$ (resp. $\widehat{g}$ ) is the sum of those monomials in the Taylor expansion of $\widehat{f}$ (resp. $\widehat{g})$ where $j /(m-k)$ is maximal, with $j$ being the power of $x$, and $k$ the power of $y$. If $\widehat{g}=$ $y^{m} V\left(x^{\alpha} / y^{\beta}\right)$ then

$$
\operatorname{Jac}(\widehat{f}, \widehat{g})=\alpha m x^{\alpha-1} y^{2 m-\beta-1}\left(V W^{\prime}-W V^{\prime}\right)\left(x^{\alpha} / y^{\beta}\right) .
$$

Thus $V=$ const. $W$.

For any non-zero polynomial $P(z, w) \in \mathcal{P}\left(\mathbb{C}^{2}(z, w)\right)$ (the set of all polynomials in $(z, w))$ we adopt the following notation:

$$
\begin{gathered}
\widetilde{P}(x, y):=P \circ(f, g)(x, y), \quad \widetilde{P}=\widehat{P}+P_{1}, \quad \text { where } \\
\widehat{P}(x, y)=x^{N} y^{M} \Phi_{P}\left(x^{\alpha} / y^{\beta}\right), \quad \Phi_{P} \text { a polynomial, } \Phi_{P}(0) \neq 0, \\
P_{1}(x, y)=\sum_{(j-N) /(M-k)<\alpha / \beta} d_{j k} x^{j} y^{k} .
\end{gathered}
$$

(To get $\widehat{P}$ we sum up those monomials $d_{j k} x^{j} y^{k}$ in the Taylor expansion of $\widetilde{P}$ for which $j+(\alpha / \beta) k$ is maximal. The monomial $x^{N} y^{M}$ is their greatest common divisor, which is guaranteed by the condition $\Phi_{P}(0) \neq 0$.)

We now define a subfamily $\mathcal{A}$ of $\mathcal{P}\left(\mathbb{C}^{2}(z, w)\right)$ by

$$
\mathcal{A}=\left\{P \in \mathcal{P}\left(\mathbb{C}^{2}(z, w)\right): \widehat{P}=\text { const. } \widehat{f}^{\varrho}, \varrho \text { a rational number }\right\}
$$

(the coefficient of $y^{m \varrho}$ in $\widehat{f}^{\varrho}$ is assumed to be 1 ). First note that the constants do belong to $\mathcal{A}$. Next we exhibit a polynomial $P_{0}$ not in $\mathcal{A}$. The image of the line $\{x=0\}$ under $\Phi$ is algebraic and hence it is the zero set of some polynomial $P_{0}(z, w)$. Since $x$ divides $\widehat{P}_{0}$ but not $\widehat{f}, P_{0}$ does not belong to $\mathcal{A}$. 
These remarks ensure the existence of a non-constant polynomial $Q(z, w)$ of the lowest degree among those from $\mathcal{P}\left(\mathbb{C}^{2}(z, w)\right)-\mathcal{A}$.

A close look at this polynomial and its partial derivative $(\partial / \partial w) Q=Q_{w}$ (which by definition of $Q$ is a member of $\mathcal{A}$ ) will help us to prove the theorem. By the chain rule we get

$$
\widetilde{Q_{w}}=(\operatorname{Jac}(f, g))^{-1} \operatorname{Jac}(f, \widetilde{Q})=c_{0} \operatorname{Jac}(f, \widetilde{Q}),
$$

where $\operatorname{Jac}(\phi, \psi)$ stands for the Jacobian of the mapping $(\phi, \psi)$. Fix $c_{1} \in \mathbb{C}$ and $\varrho \in \mathbb{Q}$ satisfying $\widehat{Q_{w}}=c_{1} \widehat{f}^{\varrho}$ and put $V:=\Phi_{Q}$. So

$$
\widehat{Q}(x, y)=x^{N} y^{M} V\left(x^{\alpha} / y^{\beta}\right) .
$$

Considering three possible cases:

$$
\text { (a) } N>1, \quad \text { (b) } N=1, \quad \text { (c) } N=0,
$$

we first check that neither (a) nor (b) can really occur, and then we show how to reduce the degree of $\Phi$ for $N=0$.

(a) Suppose $N>1$. Let us take into account only those monomials in the expansions of $\widehat{f}, \widehat{Q}$ and $\widehat{Q_{w}}$ whose $y$-degree is maximal. These are $y^{m} W(0), x^{N} y^{M} V(0)$ and $c_{1} y^{\varrho m} W^{\varrho}(0)$ respectively. Since $\operatorname{Jac}\left(W(0) y^{m}\right.$, $\left.V(0) x^{N} y^{M}\right) \neq 0$ the following equality must hold:

$$
\begin{aligned}
c_{1} y^{\varrho m} W^{\varrho}(0) & =c_{0} \operatorname{Jac}\left(W(0) y^{m}, V(0) x^{N} y^{M}\right) \\
& =-W(0) V(0) N m x^{N-1} y^{M+m-1} .
\end{aligned}
$$

This is not true for $N>1$.

(b) Suppose $N=1$. Note that $\operatorname{Jac}(f, \widetilde{Q})=\operatorname{Jac}(\widehat{f}, \widehat{Q})$ unless the right hand side is 0 . Hence with $u$ standing for $x^{\alpha} / y^{\beta}$ we may write

$$
\begin{aligned}
& \left.\left(1 / c_{0}\right) \widehat{Q_{w}}=\widehat{\operatorname{Jac}(f}, \widetilde{Q}\right) \\
= & \alpha\left(y^{m} / x\right) u W^{\prime}(u)\left(-\beta x y^{M-1} u V^{\prime}(u)+M x y^{M-1} V(u)\right) \\
& -\left(-\beta y^{m-1} u W^{\prime}(u)+m y^{m-1} W(u)\right)\left(y^{M} V(u)+\alpha y^{M} u V^{\prime}(u)\right) \\
= & y^{M+m-1}\left(-m W(u) V(u)+(\alpha M+\beta) u W^{\prime}(u) V(u)-\alpha m u W(u) V^{\prime}(u)\right) .
\end{aligned}
$$

Hence

(3) $-m W(u) V(u)+(\alpha M+\beta) u W^{\prime}(u) V(u)-\alpha m u W(u) V^{\prime}(u)=c_{2} W^{\varrho}(u)$, with $c_{2}=c_{1} / c_{0}$ and $\varrho=(M-1) / m+1$.

Set $A:=\operatorname{deg} W$ and $B:=\operatorname{deg} V$. Since $\widehat{f}=y^{m} W\left(x^{\alpha} / y^{\beta}\right)$ is a polynomial, obviously

$$
m \geq \beta A \text {. }
$$

The degree of the polynomial on the left of (3) does not exceed $A+B$. It is less than $A+B$ iff $m-A(\alpha M+\beta)+\alpha m B=0$. So either 
(i) $A+B=A \varrho=A((M-1) / m+1)$, or

(ii) $m-A(\alpha M+\beta)+\alpha m B=0$.

It is easy to check that in both cases

$$
B / A \leq M / m .
$$

Indeed, if (i) is true then $B / A=M-1 / m<M / m$, and (ii) may be rewritten in the form

$$
B / A=(\alpha M+\beta) / m \alpha-1 / A \alpha=M / m+1 / \alpha(\beta / m-1 / A),
$$

where the term in brackets does not exceed zero (see (4)). From (5) we deduce that there exists $a \in \mathbb{C}$ such that $W(a)=0$ and if $W$ (resp. $V$ ) has zero of multiplicity $\mu$ (resp. $\nu$ ) at this point then

$$
\nu / \mu \leq B / A \leq M / m \text {. }
$$

We shall prove that these inequalities lead to a contradiction. Write

$$
W(u)=(u-a)^{\mu} W_{1}(u), \quad V(u)=(u-a)^{\nu} V_{1}(u), \quad u-a=\lambda .
$$

With this notation (3) takes the form

$$
\begin{gathered}
m \lambda^{\mu+\nu} W_{1}(u) V_{1}(u)-(\alpha M+\beta) u V_{1}(u)\left(\mu \lambda^{\mu+\nu-1} W_{1}(u)+\lambda^{\mu+\nu} W_{1}^{\prime}(u)\right) \\
+\alpha m u W_{1}(u)\left(\nu \lambda^{\mu+\nu-1} V_{1}(u)+\lambda^{\mu+\nu} W_{1}^{\prime}(u)\right) \\
=c_{2} \lambda^{\mu((M-1) / m+1)}\left(W_{1}(u)\right)^{(M-1) / m+1}
\end{gathered}
$$

Hence either $\nu \alpha m-(\alpha M+\beta) \mu=0$ or the polynomial on the left hand side has zero of multiplicity $\mu+\nu-1$ at $a$ and thus $\mu+\nu-1=\mu((M-1) / m+1)$. In the former case we have

$$
\nu / \mu=(\alpha M+\beta) / \alpha m=M / m+\beta / \alpha m>M / m,
$$

in the latter

$$
\nu / \mu=(M-1) / m+1 / \mu=M / m+(1 / \mu-1 / m)>M / m
$$

(since $\mu \leq A \leq m / \beta<m$ ).

The above inequalities contradict the choice of $a$. Thus we have proved that

$$
\widehat{Q}(x, y)=y^{m} V\left(x^{\alpha} / y^{\beta}\right)
$$

Then

$$
\widehat{Q_{w}}(x, y)=c_{0} \alpha x^{\alpha-1} y^{M+m-\beta-1}\left(m W(u) V^{\prime}(u)-M W^{\prime}(u) V(u)\right) .
$$

Since on the other hand $\widehat{Q_{w}}(x, y)=c_{1} \widehat{f}^{\varrho}(x, y)=c_{1} y^{\varrho m} W^{\varrho}(u)$ it follows that $\alpha=1$.

Now we are ready to define a polynomial automorphism $\Psi$ satisfying $\operatorname{deg} \Phi \circ \Psi<\operatorname{deg} \Phi$. Take $a \in \mathbb{C}$ such that $W(a)=0$ and set

$$
\phi(x, y)=x-a y^{\beta}, \quad \psi(x, y)=y \text {. }
$$


Then $(\phi, \psi): \mathbb{C}^{2}(x, y) \rightarrow \mathbb{C}^{2}(s, t)$ is clearly an automorphism. To prove that the degree of $F:=f \circ(\phi, \psi)^{-1}$ is less than that of $f$ we may apply the Jensen formula, or to be more explicit let us define for any $c \in \mathbb{C}$

$$
\Psi_{c}(x):=\prod_{\phi_{c}(x, y)=0} f(x, y), \quad \text { where } \phi_{c}=\phi-c \psi .
$$

This function is well defined and holomorphic outside the finite set $\{x$ : $\left.(\partial / \partial y) \phi_{c}(x, y)=0\right\}$. Since it is locally bounded as well we conclude that $\Psi_{c}$ has a unique extension to an entire function. Let us estimate the growth of $\Psi_{c}$. Take $(x, y) \in\left\{\phi_{c}=0\right\} ;$ then

$$
\left|x / y^{\beta}-a\right|=\left|c y^{1-\beta}\right| \leq C_{1}|x|^{(1 / \beta)-1}+C_{2}
$$

for some positive constants $C_{1}, C_{2}$. Setting $W(u)=(u-a) W_{1}(u)$ we have for $(x, y)$ from the zero set of $\phi_{c}$

$$
\begin{aligned}
|f(x, y)| & \leq\left|y^{m}\right|\left|x / y^{\beta}-a\right|\left|W_{1}\left(x / y^{\beta}\right)\right|+\left|f_{1}(x, y)\right| \\
& \leq C_{3}\left|x / y^{\beta}-a\right||x|^{m / \beta}+C_{4}|x|^{(m-1) / \beta}+C_{5} .
\end{aligned}
$$

Combine the above two estimates to get

$$
|f(x, y)| \leq C_{6}|x|^{(m-1) / \beta}+C_{7} \quad \text { whenever } \phi_{c}(x, y)=0 .
$$

Therefore

$$
\left|\Psi_{c}(x)\right| \leq C_{8}|x|^{m-1}+C_{9} .
$$

It follows that $\operatorname{deg} \Psi_{c} \leq m-1$. By definition, the degree of $\Psi_{c}$ is equal to the number of common zeros (counting multiplicities) of $f$ and $\phi_{c}$, which is the same as the degree of $F=f \circ(\phi, \psi)^{-1}$ restricted to the line $\{s=c t\}$. So for every $c \in \mathbb{C}, \operatorname{deg} F_{\mid s=c t} \leq m-1$ and consequently $\operatorname{deg} F \leq m-1$. Since $\widehat{g}=c \widehat{f}$ the same argument works for $G:=g \circ(\phi, \psi)^{-1}$ and thus we obtain

$$
\operatorname{deg} \Phi \circ(\phi, \psi)^{-1}<m=\operatorname{deg} \Phi,
$$

which completes the proof.

Addendum. After submitting this paper the author has learned that the present result has recently been proved by R. C. Heitmann [3] in a more general setting and by a different method.

\section{References}

[1] S. S. Abhyankar, Expansion Techniques in Algebraic Geometry, Tata Inst. Fund. Research, Bombay 1977.

[2] H. Bass, E. H. Connell and D. Wright, The Jacobian Conjecture: reduction of degree and formal expansion of the inverse, Bull. Amer. Math. Soc. 7 (2) (1982), $287-330$. 
[3] R. C. Heitmann, On the Jacobian Conjecture, J. Pure Appl. Algebra 64 (1990), $35-72$.

[4] H. W. E. Jung, Über ganze birationale Transformationen der Ebene, J. Reine Angew. Math. 184 (1942), 161-174.

[5] O.-H. Keller, Ganze Cremona-Transformationen, Monatsh. Math. Phys. 47 (1939), 299-306.

[6] L. G. Makar-Limanov, On automorphisms of the free algebra on two generators, Funktsional. Anal. i Prilozhen. 4 (3) (1970), 107-108 (in Russian).

[7] K. Rusek, Polynomial automorphisms, preprint 456, IM PAN, 1989.

[8] A. G. Vitushkin, On polynomial transformations of $\mathbb{C}^{n}$, in: Manifolds, Tokyo 1973, Univ. of Tokyo Press, 1975, 415-417.

INSTITUTE OF MATHEMATICS

JAGIELLONIAN UNIVERSITY

REYMONTA 4

30-059 KRAKÓW, POLAND 